\title{
Ultra-Fast Automated TEM Electron Diffraction Tomography
}

\author{
Mauro Gemmi ${ }^{1}$, Stavros Nicolopoulos ${ }^{2}$ \\ 1. Center for Nanotechnology Innovation@NEST, Istituto Italiano di Tecnologia, Pisa Italy \\ 2. NanoMEGAS SPRL Blvd Edmond Machtens 79, B-1080 Brussels Belgium
}

The study of beam sensitive materials (zeolite, hybrid mesoporous crystals, organic crystals, pharmaceutical and proteins) with electron diffraction (ED) is a challenging task as very often those materials are difficult to be crystallized in crystals big enough/or in enough quantity to be studied with $\mathrm{X}$-Ray diffraction. Therefore, if a low dose single crystal ED technique would be available, it should be a breakthrough in crystal structure analysis. For TEM based crystal structure determination, very promising results have been obtained with the precession diffraction [1] assisted ED tomography technique [2] in which a series of patterns are collected every $1^{\circ}$ while the sample is tilted around the goniometer axis. A drawback of the technique is that after each tilt step the crystal moves, therefore a method of automation for tracking the crystal position must be developed. There is (limited to some TEM platforms) an automated version of diffraction tomography, where crystal tracking is performed in STEM mode and the diffraction is collected in nanobeam mode [3]. In this way electron radiation dose is minimized with respect to a crystal tracking based on conventional TEM imaging. One critical aspect of the latter procedure is however long data collection times, between 1 and 2 hours, due to dead time while changing between diffraction / STEM image mode and due to the CCD slow speed data collection procedure.

To speed up diffraction pattern data collection we tested at first a faster detector. As a proof of concept we collected an entire ED tomography experiment on a mayenite crystal $\mathrm{Ca}_{12} \mathrm{Al}_{21} \mathrm{O}_{33}$ by recording (tilt range $+/-60^{\circ}$ ) a total of 121 patterns on a Zeiss Libra 120 with ultra-fast Stingray CCD camera using the same experimental configuration for TEM orientation imaging technique (ASTAR) described in [4]. The Stingray CCD has 256 grey levels dynamic range and a limited number of pixels (configuration used $512 \times 512$ ) but it can record up to 100 frames/sec. For the ASTAR technique set up we used a commercially available system[5]. Since the Stingray CCD is external to the column and its optical axis is not normal to the fluorescent screen, it introduces distortions in the collected diffraction patterns. After correcting for such distortions, the patterns can be processed with the ADT3D software [3] to obtain unit cell parameters and diffraction intensities. In our experiment, the mayenite unit cell parameters have been obtained with an error of $2 \%$ and the collected ED intensities allowed the complete structure to be solved with direct methods.

As a second step we tried to automate data collection. We observed that while tilting the TEM holder, the sample always moved along a definite direction for a specific (quite large) tilt range. However the crystal movement could be tracked by shifting the beam along the same direction and the diffraction patterns could be simultaneously recorded with the Stingray CCD while working at high speed ( $>40$ frames/s). We were able to do an entire diffraction tomography data collection during the time needed by goniometer to automatically cover a large tilt range. For that we used the ASTAR control unit for TEM where it is possible to scan a rectangular area with a certain scan step (from 1 to several $100 \mathrm{~nm}$ ) and to collect an ED pattern at each point with the Stingray camera. For our purpose we defined the rectangular area elongated along the direction of crystal movement during the goniometer tilt and we have been able to track relative crystal movement for a tilt of $40^{\circ}$. A total of 362 diffraction patterns for $40^{\circ}$ of tilt have been recorded with an angular resolution of nearly $0.1^{\circ}$ where the entire data collection 
took only 3 minutes. The high angular resolution obtained in our experiment is similar to the one obtained with rotation diffraction tomography [6] and although initial data collection have been performed without precession, precession diffraction can be also be included in the experimental setup. Our analysis with ADT3D indicates that collected patterns with this setup in the $40^{\circ}$ range are enough for unit cell parameter determination, which in fact can be considered as a breakthrough due to the ultrafast data collection time, which extends the application of electron diffraction tomography method to beam sensitive materials.

\section{References:}

[1] E. Mugnaioli, T. Gorelik and U. Kolb, Ultramicroscopy 109 (2009) 758-765.

[2] R. Vincent and P.A. Midgley, Ultramicroscopy 53 (1994) 271-282.

[3] U. Kolb, T. Gorelik, C. Kübel, M. T. Otten and D. Hubert, Ultramicroscopy 107 (2007) 507-513.

[4] E.F. Rauch, J. Portillo, S. Nicolopoulos, D. Bultreys, S. Rouvimov and P.Moeck, Zeitschrift für Kristallographie: (2010) Vol. 225, issue 2-3 pp. 103-109

[5] NanoMEGAS www.nanomegas.com

[6] D. Zhang, P. Oleynikov, S. Hovmoller, X. Zou, Z. Kristallogr. 225 (2010) 94-102.

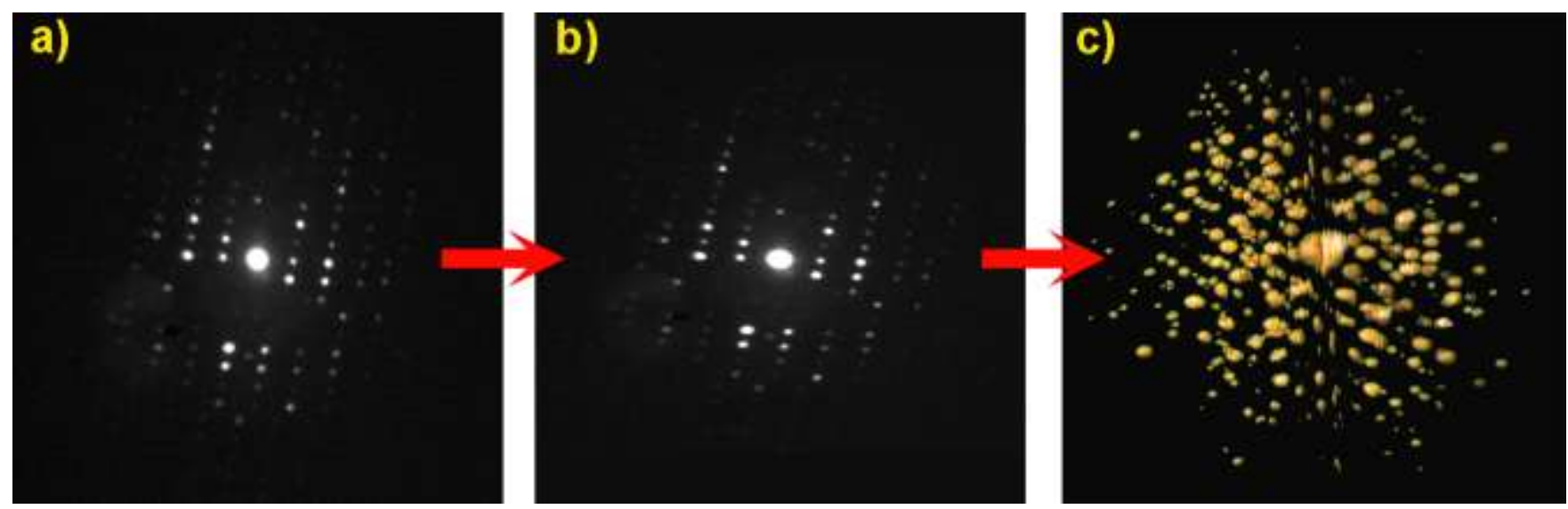

Figure 1. a) ED pattern collected on mayenite with the Stingray CCD camera. b) The same pattern after the distortion correction. c) 3D reconstruction of the reciprocal space of a mayenite crystal derived from the data collection with the Stingray camera.
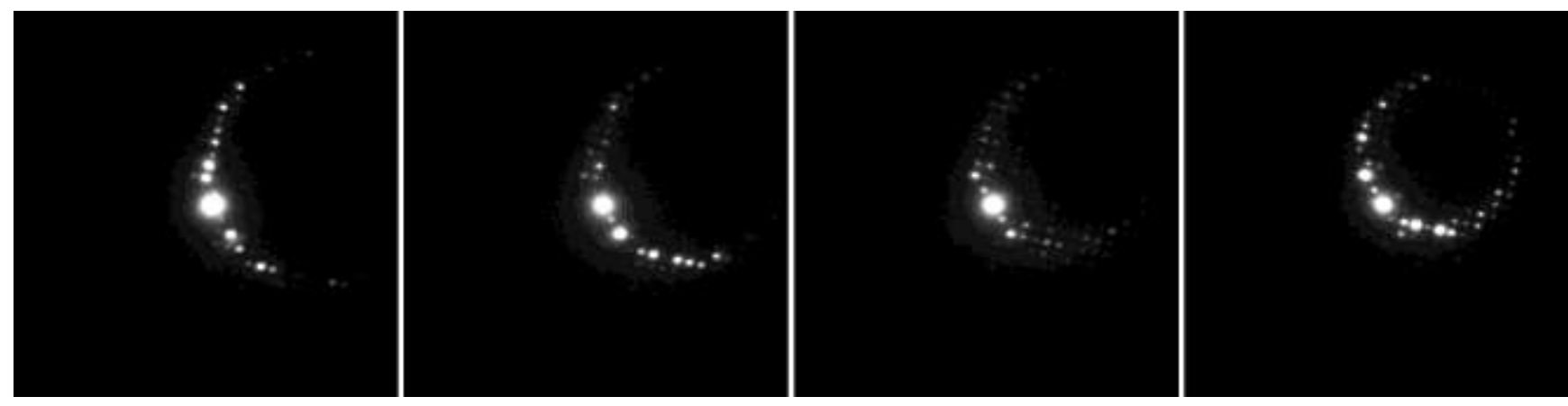

Figure 2. Sequence of ED patterns collected with the Stingray camera on mayenite crystal in the automatic mode. The angle between each pattern is $0.3^{\circ}$. 